\title{
INTERVENÇÕES ASSISTIDAS POR ANIMAIS: EFEITOS AOS CÃES TERAPEUTAS E SERES HUMANOS
}

\author{
(Animal assisted interventions: effects on therapist dogs and humans)
}

\begin{abstract}
'Camila Moura de Lima, ${ }^{1}$ Emanuele Prado Silva, ${ }^{1}$ Anne Karoline da Silveira Flores, ${ }^{2}$ Dione Moreira Nunes, ${ }^{3}$ Tatiane Morgana da Silva, ${ }^{3}$ Carolina da Fonseca Sapin, ${ }^{1}$ Fernanda Dagmar Martins Krug, ${ }^{1}$ Márcia de Oliveira Nobre

'Universidade Federal de Pelotas, Capão do Leão, Rio Grande do Sul, Brasil; ${ }^{2 E s c o l a ~ d e ~ E n s i n o ~}$ Fundamental Afonso Vizeu, Pelotas, Rio Grande do Sul, Brasil; ${ }^{3}$ Universidade Federal de Pelotas, Pelotas, Rio Grande do Sul, Brasil.
\end{abstract}

E-mail para correspondência: camila.moura.lima@hotmail.com

RESUMO - As Intervenções Assistidas por Animais utilizam os animais como facilitadores de um processo terapêutico, envolvendo a interação com diversos profissionais da área da saúde e da educação. A fim de promover o bem-estar dos coterapeutas e dos assistidos preconiza-se um rigoroso controle de higiene e saúde. O objetivo deste trabalho foi verificar a percepção dos pais/tutores e dos profissionais de educação e saúde em relação às IAAs realizadas em crianças e adolescentes e avaliar o bem-estar dos coterapeutas durantes as intervenções. As visitas ocorreram, semanalmente, com duração aproximada de 30 a 60 minutos nas instituições, INS1 e INS2, da cidade de Pelotas/RS. A análise da percepção dos pais/tutores foi realizada através de um formulário contendo dez questões objetivas abrangendo o efeito das IAAs aos assistidos e também pela avaliação inicial e final de cada assistido realizado pelos profissionais envolvidos de cada instituição. $O$ bem-estar dos cães coterapeutas foi avaliado através dos sinais vitais e da análise comportamental. Foi verificado que os assistidos apresentaram melhora na socialização, concentração na escola e em desenvolver suas tarefas, e demonstraram-se mais motivados e tranquilos nos dias das sessões. Não houve alterações nos sinais vitais e comportamentais dos cães. Conclui-se que após a análise da percepção dos pais/tutores e dos profissionais de educação e saúde foi possível verificar que as IAAs proporcionaram sensações de tranquilidade e bemestar aos assistidos, e nas condições deste estudo os coterapeutas não demonstraram sinais de desconforto e estresse durante as sessões.

Palavras-chave: coterapeuta; pet terapia; bem-estar.

ABSTRACT - Animal Assisted Interventions use animals as facilitators of a therapeutic process, involving interaction with various health and education professionals. In order to promote the welfare of therapists and assisted persons, strict hygiene and health control is recommended. The objective of this work was to verify the perception of parents / guardians and education and health professionals in relation to IAAs performed in children and adolescents and to assess the welfare of the therapists during the interventions. The visits took place weekly, lasting approximately 30 to 60 minutes at the institutions, INS1 and INS2, in the city of Pelotas / RS. The analysis of the parents / guardians perception was performed through a form containing ten objective questions covering the effect of IAAs on the 
assisted and also by the initial and final assessment of each assisted performed by the professionals involved in each institution. The welfare of the kitten dogs was assessed using vital signs and behavioral analysis. It was found that the participants showed improvement in socialization, concentration at school and in developing their tasks, and were more motivated and relaxed on the days of the sessions. There were no changes in the dogs vital and behavioral signs. It is concluded that after analyzing the perception of parents / guardians and education and health professionals, it was possible to verify that the IAAs provided feelings of tranquility and welfare to the assisted, and in the conditions of this study, the therapists did not show signs of discomfort and stress during sessions.

Keywords: cotherapist; pet therapy; welfare.

\section{INTRODUÇÃO}

As Intervenções Assistidas por Animais (IAAs) caracterizam-se pela utilização de animais como mediadores de um processo terapêutico, educacional ou como atividade lúdica. Essa prática envolve uma equipe multidisciplinar como, profissionais da saúde e educação (NOBRE, 2017). A interação entre o assistido e o cão terapeuta pode proporcionar diversos benefícios aos seres humanos como, a diminuição da ansiedade, sensação de bem-estar, melhora na comunicação e socialização, diminuição da pressão arterial, redução de estresse e diminuição da agressividade (GODOY, 2007; RIBEIRO, 2011; MOREIRA et al., 2016). O Pet terapia, da Universidade Federal de Pelotas, realiza IAAs, desde o ano de 2006, na cidade de Pelotas/RS. A equipe caracteriza-se por ser multiprofissional envolvendo docentes e discentes da graduação e pós-graduação. Os cães atuantes como coterapeutas recebem cuidados rígidos de saúde e higiene e também são capacitados e treinados, diariamente, para desenvolver as atividades nas instituições.

Os benefícios das IAAs podem auxiliar também na prevenção, melhoria e no desenvolvimento dos indivíduos como, por exemplo, em crianças com diversos tipos de deficiências. Como foi evidenciado em um estudo, que realizou uma comparação entre pacientes com transtornos psiquiátricos de idade variável, onde foi possível verificar, que no geral os pacientes apresentaram maior participação e motivação, melhorias clínicas e comportamentais significativas, melhora nas habilidades e estímulos sensoriais, aumento do tônus muscular e por fim relataram que as IAAs podem ser utilizadas como suporte para o tratamento e reabilitação de pacientes (ELMACI, 2015; STEFANINI, 2015). Já outro estudo realizado em crianças com transtorno do espectro autismo (TEA) a presença do cão terapeuta possibilitou resultados significativos referentes ao tempo de reação durante a sessão de psicoterapia, também foi possível verificar que houve uma maior interação social verificada por meio das regras sociais como a saudação e despedida (NOGUEIRA et al., 2017).

Os cães coterapeutas são cães de trabalho, portanto é importante e necessário verificar os efeitos que essa prática pode proporcionar aos cães a fim de promover e garantir o bem-estar dos coterapeutas e dos assistidos. Considerando isso esses animais necessitam de uma série de cuidados de saúde, higiene e condicionamento para participar das IAAs (CHELINI \& OTTA, 2016). Uma forma de verificar o bem-estar dos animais pode ser por meio da avaliação dos sinais vitais e da linguagem corporal (BROOM \& MOLENTO, 2004; YAMOTO, 2012). Diante ao 
exposto, é de grande relevância realizar estudos nessa área, com o intuito de inserir as IAAs como uma abordagem, que auxilie na promoção de melhorias, bem-estar e tranquilidade aos assistidos durante e após as sessões e também promover o bemestar dos coterapeutas. Desta forma, o objetivo deste trabalho foi analisar a percepção dos pais/tutores e dos profissionais de educação e saúde em relação às IAAs realizadas em crianças e adolescentes e também avaliar o bem-estar dos coterapeutas durantes as intervenções.

\section{MATERIAL E MÉTODOS}

Este trabalho trata-se de um estudo transversal, descritivo e de abordagem qualitativa. As IAAs foram desenvolvidas por uma equipe multiprofissional composta por um profissional de medicina, medicina veterinária e um profissional da educação, também havia a participação de um aluno da graduação em medicina veterinária em uma escola (INS1) e uma unidade básica de saúde (INS2). Na INS1 as IAAs foram conduzidas pelo profissional da educação e pela médica veterinária com participação de um aluno da graduação, três cães terapeutas e quatro crianças com déficit intelectual com idades entre 8 e 11 anos.

Enquanto na INS2 as atividades foram conduzidas por um profissional da medicina e medicina veterinária, um aluno da graduação em medicina veterinária, dois cães e dois pacientes um com transtorno do déficit de atenção e hiperatividade (TDAH) e outro com transtorno global de desenvolvimento (TGD), com idades entre 8 e 15 anos. Em ambos os locais as IAAs ocorreram semanalmente, totalizando 17 encontros de abril a novembro de 2018, com duração de 30 a 60 minutos, cada intervenção. Os animais foram classificados por números e distribuídos na INS1 os cães 1, 2 e 3 e na INS2 os cães 4 e 5 . O termo de consentimento livre e esclarecido foi entregue e assinado pelos pais/tutores e para as crianças o termo de assentimento livre e esclarecido.

A análise do bem-estar dos animais foi verificada pelo profissional da medicina veterinária por meio do monitoramento dos sinais vitais e da visualização da linguagem corporal dos cães. Os sinais vitais foram mensurados em dois momentos: M1 antes de ir para as instituições e M2 após o retorno das atividades. Os parâmetros mensurados em cada momento consistiram em: frequência cardíaca (FC), frequência respiratória (FR), temperatura corporal (TC), e pressão arterial sistólica (PAS). A PAS foi avaliada pelo método não invasivo, no membro posterior direito, com auxílio do aparelho digital Tech Line sendo mensurada três vezes em cada momento. Todas estas aferições foram analisadas por meio do programa Graphpadprism 7.0, pelo teste de normalidade de Shapiro-wilk e o teste t, comparando as médias obtidas em M1 e M2.

A linguagem corporal dos cinco cães foi avaliada através de filmagens individuais em cada instituição, com duração de 15 minutos. A avaliação das filmagens caracterizou-se pela observação das expressões corporais, a cada cinco minutos, através de um etograma. O etograma foi desenvolvido pela equipe do pet terapia e avalia o cão terapeuta através da linguagem corporal podendo ser dividido em categorias como, postura (de pé, sentado/deitado, empinado/colo), posição dos olhos, orelhas, cauda e cabeça, vocalizações, interação com humanos, comportamentos passivos, locomoção, interação com o ambiente, comportamentos ativos/repetitivos, comportamento típicos de estresse (ROSA, 2015; SANTOS, 2017). Com base nisso, os avaliadores da equipe observaram a linguagem corporal dos cães coterapeutas durante as atividades, para classificar condições de estresse ou de conforto, considerando a posição das orelhas, boca, cabeça e cauda, a 
expressão do olhar e a postura corpórea durante as atividades (Tabela 1), representadas na Figura 1.

Tabela 1- Parâmetros avaliados nos cães terapeutas durante as IAAs.

\begin{tabular}{|c|c|c|c|}
\hline Parâmetros & Bem-estar & & Estresse ou agressividade \\
\hline Orelhas & Relaxadas e/ou em alerta & & Voltadas para trás ou para baixo \\
\hline Olhos & Relaxados & & Em alerta e/ou indireto \\
\hline Boca & Aberta relaxada & & Fechada rígida \\
\hline Cabeça & $\begin{array}{l}\text { Levantada sem sinal } \\
\text { tensão }\end{array}$ & de & Baixa com sinais de tensão \\
\hline Postura & Em pé e/ou deitado & & Curvada/tensa \\
\hline Cauda & $\begin{array}{l}\text { Relaxada, em } \\
\text { abanando }\end{array}$ & ou & Tensa e/ou entre as pernas \\
\hline
\end{tabular}

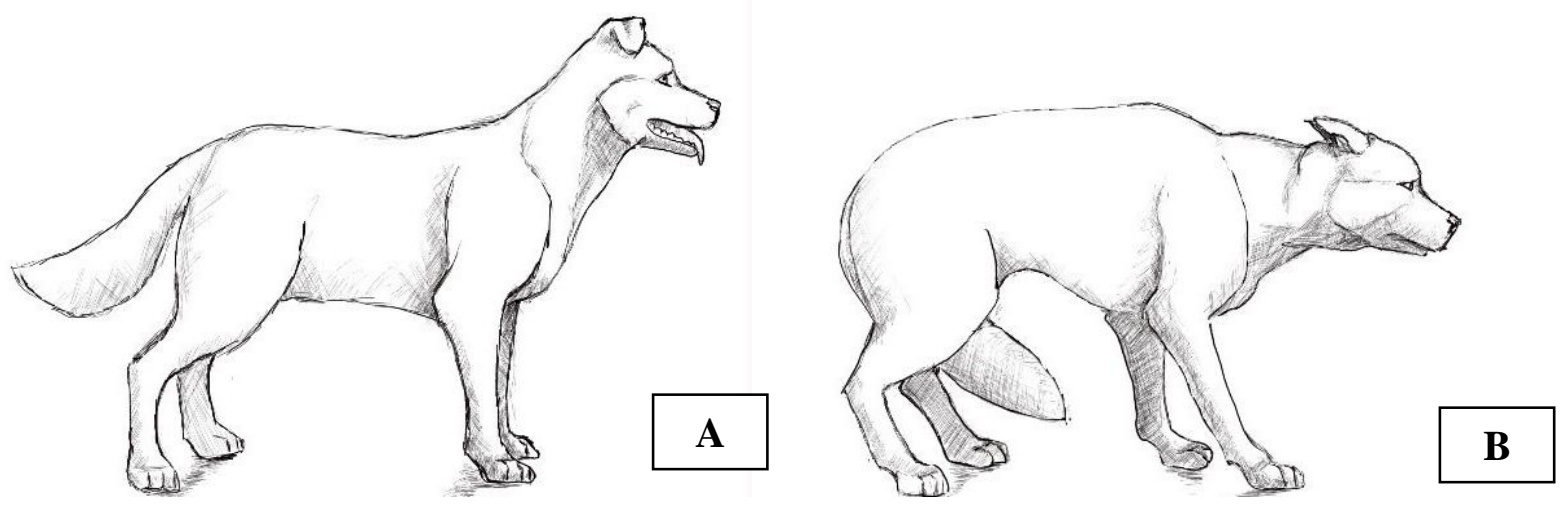

Figura 1- Imagens de cães que representam tranquilidade/Bem-estar (A) e desconforto/estresse(B). Imagens obtidas do etograma utilizado no pet terapia.

Durante as IAAs a equipe buscou estabelecer o vínculo entre o assistido e o coterapeuta a fim de promover uma aproximação entre ambos, por meio o toque e carinho. Após o estabelecimento do vínculo foi desenvolvida uma série de atividades conduzidas pelos profissionais da saúde e educação tendo como temática o cão terapeuta tanto na forma presencial como na forma de materiais lúdicos (bonecos e jogos). No decorrer das IAAs procurou-se trabalhar com atividades que estimulassem a afetividade, a motricidade e a cognição dos assistidos. Dentre as atividades realizadas, utilizou-se o colete pedagógico (Figura 2), que além de promover o contato e o vínculo com o coterapeuta, possibilitou o desenvolvimento de diversas habilidades, usando letras, números, cores, formas, além de estimular movimentos com a abertura e fechamento de zíper e passagem de fios em ilhoses. 


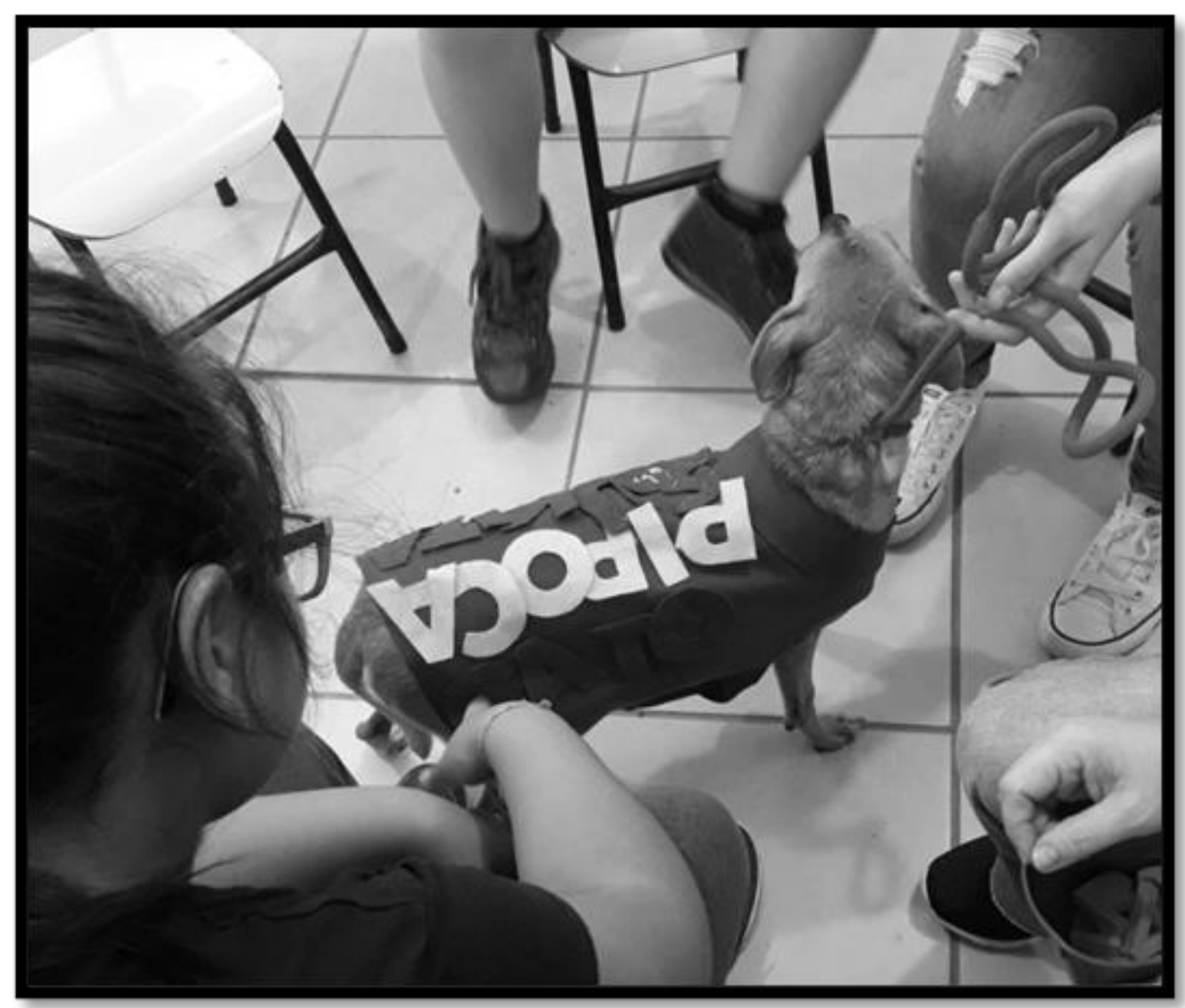

Figura 2 - Atividades na sala de recurso com a utilização do colete pedagógico.

A análise da percepção dos pais/tutores em relação aos efeitos das IAAs aos assistidos foi realizada pelos profissionais de cada instituição após dois semestres de atividades por meio a aplicação de um formulário os tutores/pais de cada assistido contendo dez questões objetivas, que abrangeram perguntas como, se o assistido possuía animal de estimação, se houve uma melhora na afetividade, socialização, concentração e execução das brincadeiras após as intervenções e se o pai/tutor acreditava que as atividades poderiam contribuir positivamente para 0 assistido, Tabela 3. Também foi realizado um relato final da percepção dos efeitos das IAAs aos assistidos por cada profissional da INS1 e INS2.

\section{RESULTADOS E DISCUSSÃO}

De acordo com os resultados obtidos na análise do bem-estar dos cães terapeutas foi possível verificar que não houve alterações significativas $(P>0,05)$ nos valores da frequência cardíaca e respiratória, pressão arterial sistólica e temperatura corporal durante o trabalho realizado nas IAAs (Tabela 2). Esses resultados podem ser explicados porque os cães do pet terapia são capacitados e treinados diariamente para realizar as atividades nas instituições para que o manejo e o trabalho realizado durante as IAAs estejam dentro da rotina de cada um dos cães e sejam entendidas por eles como motivadoras e dentro das condições de bem-estar. Dessa forma, contribuindo para os resultados obtidos neste estudo. 
A realização de atividade física e o transporte até o local de visitação podem gerar aumentos significativos nos sinais vitais dos cães de trabalho (ROVIRA, 2008; CHELINI \& OTTA, 2016). Assim os cães terapeutas devem ser condicionados e adaptados a uma série de situações como a socialização com seres humanos e outros animais, dessensibilização ao toque, treinamento, adaptação ao transporte e a ambientação ao ambiente de trabalho (CHELINI \& OTTA, 2016).

Tabela 2- Média dos sinais vitais dos cães coterapeutas pré e pós atividades nas instituições 1 e 2 sendo avaliada a frequência cardíaca e respiratória, pressão arterial sistólica e temperatura corporal.

\begin{tabular}{lllllllllll}
$\begin{array}{c}\text { Sinais Vitais } \\
\text { (Valores fisiológicos) }\end{array}$ & \multicolumn{4}{c}{ MÉdIA INS1 } & \multicolumn{4}{c}{ MÉdIA INS2 } \\
& CÃO 1 & \multicolumn{1}{c}{ CÃO 2 } & \multicolumn{1}{c}{ CÃO 3 } & CÃO 4 & CÃo 5 \\
\hline MOMENTO & Pré & Pós & Pré & Pós & Pré & Pós & Pré & Pós & Pré & Pós \\
FC $(60-160 \mathrm{bpm})$ & 80 & 83 & 96 & 94 & 92 & 86 & 88 & 83 & 89 & 93 \\
FR $(18-36 \mathrm{mpm})$ & 26 & 24 & 34 & 24 & 40 & 34 & 22 & 22 & 38 & 41 \\
PAS $(120-152 \mathrm{mmhg})$ & 158 & 160 & 138 & 125 & 132 & 151 & 98 & 109 & 142 & 131 \\
TC $\left(37,4-39,2^{\circ} \mathrm{C}\right)$ & 38 & 39 & 37 & 38 & 37 & 38 & 38 & 38 & 37 & 38 \\
\hline
\end{tabular}

Legenda: bpm= batimento por minuto; $\mathrm{mpm}=$ movimento por minuto; $\mathrm{mmhg}=$ milímetro de mercúrio; ${ }^{\circ} \mathrm{C}=$ grau Celsius

Após a análise da linguagem corporal dos coterapeutas (1,2 e 3 ) que atuaram na INS1, foi possível identificar que os cães 1, 2 e 3 permaneceram a maior parte do tempo oscilando em estar em pé e sentado, olhos e orelhas relaxados, uma parte do tempo observando as pessoas e interagindo com os assistidos.

O cão 1 e 2 estendeu a pata solicitando carinho dos assistidos, o cão 3 interagiu com os assistidos, mostrando tranquilidade e satisfação, com a cauda abanando e deslocou-se quando solicitado. Na INS2 os cães 4 e 5 permaneceram a maior parte do tempo em pé, olhos e orelhas relaxadas, cauda e cabeça levantadas, ficaram atentos observando os membros da equipe e os pacientes e deslocaram-se quando solicitados para realizar os passeios com os assistidos. Os cães interagiram com os pacientes durante as atividades, sendo que o cão 5 estendeu a pata e o cão 4 demonstrou-se curioso cheirando o ambiente.

Em ambas instituições não foram observadas alterações da linguagem corporal que determinassem desconforto, estresse, cansaço, que seriam manifestados com comportamentos passivos e repetitivos. Observou-se que os cães estavam tranquilos, interagindo e mantendo o olhar com os assistidos, manifestando alegria $e$ atentos ao local $e$ as pessoas presentes. $O$ bem-estar pode estar correlacionado com a capacidade de resiliência que o animal possui se adaptando ao ambiente que está inserido (BEERDA, 1997). Com isso, de uma maneira geral o objetivo a ser alcançado com os pacientes durante as IAAs, o tipo de assistido e a duração das IAAs podem promover alterações fisiológicas e/ou comportamentais nos cães terapeutas (ROSA, 2015).Também há outros fatores que podem promover situações estressantes como, por exemplo, barulhos externos e o período de transporte até o local de visitação (BEERDA, 1997). Assim tanto as situações de felicidade, como medo e até mesmo agressividade são manifestadas através da linguagem corporal (COLLINS, 2009). 
A avaliação dos assistidos foi verificada através da aplicação de um formulário aos tutores/pais dos assistidos $A, B, C, D$ na INS1 e E, F na INS2. Conforme verificado na (Tabela 3), onde percebe-se que houve melhora na relação com os familiares e animais de estimação, as atividades proporcionaram mais tranquilidade e motivação aos assistidos e também verificou-se melhora na socialização e concentração na atividades escolares.

Tabela 3 - Avaliação do formulário aplicado aos tutores/pais dos assistidos A, B, C, D na INS1 e E, F na INS2.

\begin{tabular}{|c|c|c|c|c|c|c|}
\hline \multirow[t]{2}{*}{ Questões } & \multicolumn{4}{|c|}{ INS1 } & \multicolumn{2}{|c|}{ INS2 } \\
\hline & A & B & C & $\mathbf{D}$ & $\mathbf{E}$ & $\mathbf{F}$ \\
\hline Possuía animal de estimação? & $x$ & - & - & $X$ & - & $X$ \\
\hline $\begin{array}{l}\text { Notou melhora na relação com } \\
\text { o animal de estimação? }\end{array}$ & ++ & +++ & ++ & ++ & + & +++ \\
\hline $\begin{array}{l}\text { Notou melhora com os } \\
\text { familiares? }\end{array}$ & + & +++ & ++ & 0 & + & + \\
\hline $\begin{array}{l}\text { O assistido demonstra estar } \\
\text { mais tranquilo após as } \\
\text { atividades? }\end{array}$ & +++ & +++ & +++ & 0 & ++ & ++ \\
\hline $\begin{array}{l}\text { Notou melhora na socialização } \\
\text { com outras pessoas? }\end{array}$ & ++ & +++ & +++ & 0 & ++ & ++ \\
\hline $\begin{array}{l}\text { Percebeu melhora na } \\
\text { concentração na escola? }\end{array}$ & ++ & +++ & ++ & 0 & ++ & + \\
\hline $\begin{array}{l}\text { Acredita que o assistido sente- } \\
\text { se mais motivado nos dias de } \\
\text { Pet terapia? }\end{array}$ & ++ & +++ & +++ & 0 & ++ & +++ \\
\hline Acredita que o pet terapia pode & & & & & & \\
\hline $\begin{array}{l}\text { contribuir para } \\
\text { desenvolvimento do seu filho? }\end{array}$ & +++ & +++ & ++ & +++ & +++ & ++ \\
\hline
\end{tabular}

Legenda: $\quad x=$ sim; -= não; += leve; ++=moderado; +++= acentuado; $0=$ não se aplica

Também foi possível verificar que os tutores/pais deixaram um pequeno relato voluntariamente após a última pergunta do formulário. Dessa forma, na INS1 o familiar do aluno A relatou, que [...]as IAAs podem contribuir positivamente, pois ajuda a demonstrar afetos com os animais, o que para ele era difícil, pois possuía medo de animais[...].O familiar do aluno $B$ relatou, que o assistido também realizava equoterapia e [...] ele sente-se mais seguro na presença de cães, menos ansioso e mais corajoso. Acredito que a Pet Terapia Ihe proporcionou essa melhora, pois antes havia um 'pânico' quando chegava próximo a um cachorro e agora ele se aproxima com naturalidade e paciência [...]. A família do aluno $\mathrm{C}$ verificou, que [...] deixa ela 
mais feliz e calma [...]. Os familiares do assistido $D$ relataram, que apesar da participação do assistido ser recente já foi possível verificar, que [...] Ela já apresenta menos receio em se aproximar dos gatos em casa e as IAAs podem contribuir positivamente, porque desenvolvem habilidades de socialização e interação [...]. Na INS2 o tutor do assistido $E$ relatou, que [...] ela gosta de conversar, brincar e se relacionar com o cachorro do pet terapia [...].

De acordo com os dados dos formulários mencionados acima foi possível analisar que as atividades propostas proporcionaram diversos benefícios aos assistidos. Nesse contexto, os animais participaram como catalisadores de emoções, facilitando assim a socialização e a aprendizagem. Dessa forma, os resultados obtidos podem contribuir para uma melhora no âmbito familiar, social e afetivo do assistido (MENDONÇA, 2014).

A percepção de sensações de satisfação e tranquilidade podem estar relacionadas a liberação de hormônios a partir do contato dos assistidos com os cães durante as IAAs levando ao aumento das concentrações plasmáticas de endorfinas e no decréscimo da concentração plasmática de cortisol, ou seja, contribuindo para liberação de hormônios que proporcionam emoções de conforto e relaxamento (ODENDAAL, 2003; HARPER et al., 2015).

O profissional da educação, na INS1, relatou que os alunos tiveram motivação em realizar as atividades, afetividade pelos animais e pelos profissionais envolvidos. Demonstraram comunicação e participação nas tarefas propostas, com maior concentração e foco. Dessa forma, apresentando avanços significativos na parte pedagógica e de convívio. Também foi possível observar que os assistidos demonstraram conhecimento e interesse sobre os animais e em relação aos cuidados com os mesmos. A utilização de animais no âmbito pedagógico surgiu como uma importante ferramenta educativa. O uso de recursos lúdicos como, por exemplo, os jogos é um instrumento, que possibilita unir diversas situações mentais como, estimulação do pensamento, ordenação do tempo e espaço (CHELINI \& OTTA, 2016).

Já o profissional da INS2 observou que durante o período das atividades os assistidos demonstraram-se mais afetivos com a equipe e o cão terapeuta possibilitando, dessa forma, o desenvolvimento de algumas atividades com o colete pedagógico e demonstraram interesse em continuar participando das IAAs no próximo ano. Um ponto relevante para o desenvolvimento da IAA é o estabelecimento de vínculos, que pode ser observado através de uma maior cumplicidade e demonstração de afeto (DOTTI, 2005). Desse modo, foi possível analisar a importância desse elo entre o paciente e o coterapeuta, isso contribuiu para uma maior facilidade em desenvolver as atividades. Diante do exposto, é de grande importância realizar estudos nessa área, a fim de promover bem-estar aos assistidos e cães terapeutas.

\section{CONCLUSÃO}

Conclui-se que após a análise da percepção dos pais/tutores e dos profissionais de educação e saúde foi possível verificar que as IAAs proporcionaram sensações de tranquilidade e bem-estar aos assistidos. Também foi possível observar, que os coterapeutas não demonstraram sinais de desconforto e estresse durante as sessões, preservando, dessa forma, o bem-estar dos coterapeutas e assistidos. 


\section{NOTAS INFORMATIVAS}

O estudo foi aprovado pelo comitê de ética em experimentação animal CEEA (9431) e pela plataforma Brasil (01484818.8.0000.5316).

\section{REFERÊNCIAS}

BEERDA, B.; SCHILDER, M.B.H.; VAN HOOFF JARAM, D.V.H.W. Manifestation of chronic and acute stress in dogs. Applied Animal Behaviour Science, n.52, v. 3-4, p.307-319, 1997.

BROOM, D.M.; MOLENTO, C.F.M. Bem-estar animal: conceito e questões relacionadas- revisão. Archives of Veterinary Science, n.2, v.9, p.1-11, 2004.

CHELINI, M.O.M.; OTTA, E. Terapia assistida por animais. São Paulo: Manole, 2016. 364p.

COLLINS, S. Cachorros falam: entenda a linguagem corporal dos cães. Rio de Janeiro: Ediouro, 2009. 125p.

DOTTI, J. Terapia e Animais. São Paulo: Noética, 2005.294p.

DUARTE, M. T., NOBRE, M., RODRIGUEZ, R., SZORTYKA, A. L., KRUG, F., GÖRGEN, E., KRAMER, A. R., SANTOS, V., HERTZBERG, J., SEVERO, T., BRESOLIN, S., SILVA, R., SCHUSTER, J., PEREZ, C., HEEMANN, I., \& SANTOS, F. O cão como aspecto motivador de crianças com transtorno do espectro autismo. Revista De Estudios E Investigación En Psicología Y Educación, n.1, p. 280-283, 2017.

Elmaci, D.T.; Cevizci, S. Dog-Assisted therapies and activities in rehabilitation of children with cerebral palsy and physical and mental disabilities. International Journal of Environmental Research and Public Health, v.12, n.5, p.5046-5060, 2015.

FEITOSA, F.L.F. Semiologia veterinária a arte do diagnóstico. Rio de Janeiro: Rocca, 2014.1406p.

GODOY, A. C.; DENZIN, S. S. Atividades assistidas por animais: aspectos revisivos sob um olhar pedagógico. 2007. Acessado em 15 nov. 2018. Disponívelem:http://www.researchgate.net/publication/266328629 Atividades assisti das por animais_aspectos_revisivos_sob_um_olhar_pedaggico.

HARPER, C.M.; DONG, Y.; THORNHILL, T.S.; WRIGHT, J.; READY, J.; BRICK, G.W.; DYER, G. Can Terapy dogs improve pain and satisfaction after total joint arthroplasty? A randomized controlled trial. Clinical orthopaedics and related research, v.473, n. 1, p.372-379.

MEDONÇA, M. E.F.; SILVA, R, R.; FEITOSA, M.J.S.; PEIXOTO, S. P.L. A Terapia Assistida por cães no desenvolvimento sócio afetivo de crianças deficiência intelectual. Cadernos de graduação- Ciências biologias e da Saúde, v.2, n.2, p.11-30, 2014. 
MEURS, K.M. et al. Arterial blood pressure measurement in a population of healthy geriatric dogs. Journal of the American Animal Hospital Association, v.36, p.497-500, 2000.

MOREIRA, R.L.; GUBERT, F.A.; SABINO, L.M.M.; BENEVIDES, J.L.; TOMÉ, M.A.B.G.; MARTINS, M.C.; BRITO, M.A. Terapia assistida com cães em pediatria oncológica: percepção de pais e enfermeiros. Revista Brasileira de Enfermagem, v.69, n. 6 , p. $1168-1194,2016$

NOBRE, Márcia de Oliveira; KRUG, Fernanda Dagmar Martins; CAPELLA, Sabrina de Oliveira; RIBEIRO, Viviane Pereira; NOGUEIRA, Maria Teresa Duarte; CANIELLES, Carla; TILLMANN, Mariana Teixeira. Projeto Pet Terapia: Intervenções Assistidas por Animais: Uma prática para o benefício da saúde e educação humana. Expressa Extensão, Pelotas, v.22, n.1, p.78-89, jan-jun.2017. Disponível em: <https://periodicos.ufpel.edu.br/ojs2/index.php/expressaextensao/article/viewFile/109 21/7417>. Acesso em: 17 dez.2018.

NOGUEIRA, M.T.D.; NOBRE, M.O.; RODRIGUEZ, R.C.M.C.; SZORTYKA, A.L.S.C.; FRUG, F.; GÖRGEN, E.S..; KRAMER, A.R.B.; SANTOS, V.G.; HERTZBERG, J.C.; SEVERO, T.S.; BRESOLIN, S.D.; SILVA, R.M.L.; SCHUSTER, J.G.; PEREZ, C.C.; HEEMANN, I.M.; SANTOS, F.B.P. O cão como aspecto motivador de crianças com transtorno do espectro autismo. Revista de estudios e investigación en psicologia y educación, v.extr., n.1, p.280-283, 2017.

ODENDAAL, J.S.J.; MEINTJES, R.A. Neurophysiological correlates of affiliative behaviour between humans and dogs. The Veterinary Journal, v.165, n.3, p.296301, 2003.

RIBEIRO, A. F. A. Cães domesticados e os benefícios da interação. Revista Brasileira de Direito animal, Salvador, v. 8, n.q, p. 249-262, 2011.

ROSA, P.D.E.; RAINHO, M.R.G.; PEREIRA, G.G. Revisão sobre ética e bem-estar nas intervenções assistidas por cães. Clínica Veterinária, anoXX, n.116, p., 2015.

ROSA, Pedro David Esteves; RAINHO, Maria do Rosário Grou; PEREIRA, Gonçalo da Graça. Revisão sobre ética e bem-estar nas intervenções assistidas por cães. Revista Clínica Veterinária, São Paulo, v.116, n.20, p.40-47, maio-jun. 2015. Disponível em: < http://www.revistaclinicaveterinaria.com.br/edicao/2015/maiojunho.html>. Acesso em: 15 dez.2018.

ROVIRA, S.; BENITO, A.M.M. Effect of exercise on physiological, blood and endocrine parameters in search and rescue -trained dogs. Veterinarni Medicina, v.53, n.6, p. 333-346, 2008.

SANTOS, A.; ALVES, J.; GALHARDO, L.; FRAGOSO, S.; GONÇALO, G-P. Efeitos do enriquecimento ambiental e da coleira $D A P$ em cães de polícia. Veterinary medicine, Nov, dez., p. 30-36, 2015.

Stefanini, M.C.; Martino, A.; Allori, P.; Galeotti, F.; Tani, F. The use of Animal Assisted Therapy in adolescents with acute mental disorders: A randomized 
controlled study. Complementary Therapies in Clinical Practice, v, 21, n.1, p.4246, 2015.

VIVALVINI, V.H. Terapia Assistida por Animais: uma abordagem lúdica em reabilitação clínica de pessoas com deficiência intelectual. [Dissertação de Mestrado]. São Bernardo do Campo, SP: Universidade Metodista de São Paulo, 2011.

YAMAMOTO, K.C.M.; SILVA, E.Y.T.; COSTA, K.N.; SOUZA, M.S.; SILVA, M.L.M.; ALBUQUERQUE, V.B.; PINHEIRO, D.M.; BERNABÉ, D.G.; OLIVA, V.N.L.S. Avaliação fisiológica e comportamental de cães utilizados em terapia assistida por animais (TAA). Arquivo Brasileiro de Medicina Veterinária e Zootecnia, v.64, n.3, p.568-676, 2012. 